\title{
Serial Vagus Nerve Stimulation Functional MRI in Treatment-Resistant Depression
}

\author{
Ziad Nahas*,',2, Charlotte Teneback', Jeong-Ho Chae',3, Qiwen Mu', Chris Molnar', Frank A Kozel', \\ John Walker', Berry Anderson', Jejo Koola', Samet Kose', Mikhail Lomarev ${ }^{1,2,4}$, Daryl E Bohning ${ }^{2}$ and \\ Mark S George ${ }^{1,2,5}$
}

'Department of Psychiatry, Brain Stimulation Laboratory, Mood Disorders Program, Institute of Psychiatry, Charleston, SC, USA; ${ }^{2}$ Department of Radiology, Radiology and Center for Advanced Imaging Research, Medical University of South Carolina, Charleston, SC, USA; ${ }^{3}$ Department of Psychiatry, Catholic University, Seoul, South Korea; ${ }^{4}$ National Institute of Neurological Disorders (NINDS), Bethesda, MD, USA; ${ }^{5}$ Department of Psychiatry, Ralph H. Johnson VA Medical Center, Charleston, SC, USA

\begin{abstract}
Vagus nerve stimulation (VNS) therapy has shown antidepressant effects in open acute and long-term studies of treatment-resistant major depression. Mechanisms of action are not fully understood, although clinical data suggest slower onset therapeutic benefit than conventional psychotropic interventions. We set out to map brain systems activated by VNS and to identify serial brain functional correlates of antidepressant treatment and symptomatic response. Nine adults, satisfying DSM-IV criteria for unipolar or bipolar disorder, severe depressed type, were implanted with adjunctive VNS therapy (MRI-compatible technique) and enrolled in a 3-month, doubleblind, placebo-controlled, serial-interleaved VNS/functional MRI (fMRI) study and open 20-month follow-up. A multiple regression mixed model with blood oxygenation level dependent (BOLD) signal as the dependent variable revealed that over time, VNS therapy was associated with ventro-medial prefrontal cortex deactivation. Controlling for other variables, acute VNS produced greater right insula activation among the participants with a greater degree of depression. These results suggest that similar to other antidepressant treatments, BOLD deactivation in the ventro-medial prefrontal cortex correlates with the antidepressant response to VNS therapy. The increased acute VNS insula effects among actively depressed participants may also account for the lower dosing observed in VNS clinical trials of depression compared with epilepsy. Future interleaved VNS/fMRI studies to confirm these findings and further clarify the regional neurobiological effects of VNS.
\end{abstract}

Neuropsychopharmacology (2007) 32, 1649-1660; doi: I 0. I038/sj.npp. I 30 I288; published online 3 January 2007

Keywords: vagus nerve stimulation; $\mathrm{MMRl}$; depression; antidepressant

\section{INTRODUCTION}

Depression is a major public health problem. Approximately, $60 \%$ of patients treated with antidepressants do not achieve remission (O'Reardon and Amsterdam, 1998; Fagiolini and Kupfer, 2003). Neuroimaging studies have described the correlates of therapeutic interventions in an effort to better understand the neurobiological characteristics associated with clinical response. Although this area is relatively new and findings differ, many studies suggest that limbic and frontal brain regions change over time during successful treatment of depression regardless of the therapy.

Portions of this work were presented at the annual meeting of the American College of Neuropsychopharmacology, San Juan, Puerto Rico, December 2004.

*Correspondence: Dr Z Nahas, Brain Stimulation Laboratory, Mood Disorders Program, Institute of Psychiatry, 67 President Street, Room 502 North, Charleston, SC 29403, USA, Tel: + I 8437925710 , Fax: + I 843792 5702, E-mail: nahasz@musc.edu

Received 2 January 2006; revised 19 October 2006; accepted 24 October 2006
Such changes have been found with sleep deprivation $(\mathrm{Wu}$ and Bunney, 1990; Ebert et al, 1991, 1994; Wu et al, 1992), medications (Buchsbaum et al, 1997; Mayberg et al, 2000; Nobler et al, 2000; Drevets et al, 2002), placebo (Kleinschmidt et al, 1999), electroconvulsive therapy (ECT) (Nobler et al, 2001), transcranial magnetic stimulation (TMS) (Teneback et al, 1999; Nahas et al, 2001b), deep brain stimulation (DBS) (Mayberg et al, 2005), cognitive behavioral therapy (Goldapple et al, 2004), or interpersonal therapy (Brody et al, 2001).

Vagus nerve stimulation (VNS) is approved by the US Food and Drug Administration (FDA) to treat refractory partial-onset seizures and, more recently, treatment-resistant depression. Antidepressant VNS effects were initially found in an open acute study (Rush et al, 2000; Sackeim et al, 2001) and long-term follow-up (Marangell et al, 2002; Nahas et al, 2005). Despite lack of a significant effect greater than placebo in a double-blind acute study (Rush et al, 2005a), of which this study was a part, the naturalistic 1- or 2-year follow-up study of adjunctive VNS therapy in two independent cohorts (D01 (Nahas et al, 2005) and D02 
(George et al, 2005; Rush et al, 2005b)) showed the antidepressant response increasing over time with initial improvements largely sustained. Long-term results showed delayed and consistent improvement with substantially lesser relapse rates than other studies of similarly treatment-resistant subjects (Prudic et al, 2004). The mechanisms of action underlying these intriguing VNS response patterns remain unexplained.

Chae et al (2003) reviewed single photon emission computed tomography (SPECT) (Ring et al, 2000; Vonck et al, 2000; Van Laere et al, 2002; Barnes et al, 2003) and positron emission tomography (Garnett et al, 1992; Ko et al, 1996; Henry et al, 1998, 1999) investigating the acute and/or chronic effects of VNS for epilepsy and depression. A recent SPECT study suggested that 4 weeks of active VNS therapy in depression is associated with decreased activity in hippocampus and amygdala and increased activity in left prefrontal cortex (Zobel et al, 2005). Researchers have also used functional MRI (fMRI) to investigate VNS in epilepsy (Narayanan et al, 2002; Sucholeiki et al, 2002; Liu et al, 2003) and depression (Maniker et al, 2000; Bohning et al, 2001; Lomarev et al, 2002; Mu et al, 2004). Initial studies of depressed participants showed the feasibility of performing VNS-synchronized fMRI studies and compared the location and amount of blood oxygenation level dependent (BOLD) signal change caused by acute VNS for $7 \mathrm{~s}$ with the period when the device was not firing (Bohning et al, 2001). At least five adjustable parameters likely contribute to the immediate- and longer-term effects of VNS: intensity, frequency, pulse width, 'on' time, and 'off time. Recent fMRI studies have demonstrated that the frequency (Lomarev et al, 2002) and pulse width(Mu et al, 2004) of VNS delivered to depressed adults produces dose-dependent modulatory effects on acute brain activity (eg, less than a minute). Little is known, however, about neurobiological effects of VNS over time beyond a period of 3 months (Henry et al, 2004) and dynamic regional brain activity changes that may correlate with depressive symptoms' severity. We used interleaved VNS/fMRI to serially scan depressed participants in a VNS clinical trial investigating how VNS parameters affect global and regional brain activity as a function of disease severity and time (Harrison et al, 2003).

\section{METHODS}

This study investigated the effects of adjunctive VNS on BOLD response over time among participants at the Medical University of South Carolina (MUSC) in a double-blind acute and open long-term follow-up clinical trial (D-02) for treatment-resistant depression (Rush et al, 2005a). Participants had not responded adequately to at least 2 , but not more than 6 , research-qualified medication trials of different antidepressant classes. All VNS-implanted outpatients enrolled at MUSC $(n=18)$ were approached for this serial VNS/fMRI study; one declined because of claustrophobia. All 17 others with either non-psychotic major depressive disorder $(n=14)$ or non-psychotic, depressed-phase bipolar disorder $(n=3)$ gave written informed consent approved by the Institutional Review Board for Human Research.

\section{Clinical Assessments}

All scanning sessions coincided with scheduled D-02 clinical trial protocol visits. Before the MRI scans on the same day, raters blinded to VNS active/placebo assignment conducted assessments, including the Number of Failed Antidepressants in current depressive episode (NFAD) at baseline according to the Antidepressant Treatment History Form (Sackeim, 2001) and the 24-item Hamilton Depression Rating Scale $\left(\mathrm{HRSD}_{24}\right)$ (Hamilton, 1967), the primary clinical efficacy outcome for acute (Rush et al, 2005a) and long-term follow-up (Rush et al, 2005b). The reliability of the placebo condition was high as described by Rush et al $(2005 a, b)$ despite a lack of physical sensation (Table 1$)$.

\section{Experimental Design and Procedure}

The VNS/fMRI study was initiated simultaneously with clinical randomization of the D-02 trial. To allow for recovery from surgery, VNS was initiated 2 weeks after implantation of the MRI-compatible device (Cyberonics, Houston, TX) (Bohning et al, 2001). The VNS study involved two phases, the 10 -week, randomized, controlled, masked trial during which concomitant medications were stable and participants were scanned three times: randomization and initial exposure to VNS (baseline); 2 weeks after VNS intensity adjustment ( $\sim$ week 2$)$; and 8 weeks later ( $\sim$ week 10$)$. The second phase was an open-label follow-up study with scans at week 13, week 20), then quarterly (see study design in Figure 1).

In the MRI suite, and before participants entered the scanner, the unblinded VNS programmer for the clinical study (see Rush et al (2005a)) changed each subjects' VNS parameters from their ongoing settings to adapt to the imaging block design paradigm (pulse width $500 \mathrm{~ms}$, pulse frequency $20 \mathrm{~Hz}$, and duty cycle of $13.6 \mathrm{~s}$ 'on' (including $3.3 \mathrm{~s}$ ramp-up and $3.3 \mathrm{~s}$ ramp-down and $41 \mathrm{~s}$ 'off'). These settings were held constant throughout the imaging, about $1 \mathrm{~h}$. The output current was maintained at the same level as the participant had been receiving except for the scan at baseline when the output current was set to a 'tolerable' level or $0 \mathrm{~mA}$ for those randomized to placebo.

Before and after each functional scan, the VNS generator signal was checked, which allowed synchronization of the fMRI scanning cycle with the VNS generator cycle (for a detailed method refer to Bohning et al (2001)).

All MRI scanning was performed using a $1.5 \mathrm{~T}$ clinical MRI scanner with a send-receive head coil (Intera, Philips Medical Systems, Bothell, WA, USA). A survey scan ascertained head location for subsequent anatomical and functional scans. A set of T1-weighted sagittal structural images encompassing the whole brain were acquired using the following parameters, $\mathrm{TR}=625 \mathrm{~ms}, \mathrm{TE}=20 \mathrm{~ms}$, slice thickness $=5 \mathrm{~mm}$, gap $=1 \mathrm{~mm}$, field of view $=256 \mathrm{~mm}$, number of slices $=27$, matrix $=256 \times 256$. Using the same slice coverage as the structural scans, a whole brain gradient echoplanar imaging (EPI) sequence was obtained for each participant, and employed the same scanning and reconstruction parameters as the structural scan except for a TR of $2837 \mathrm{~ms}$, a TE of $45 \mathrm{~ms}$, and a $128 \times 128$ matrix, resulting in a voxel size of $2 \times 2 \times 6 \mathrm{~mm}^{3}$. The functional scanning session consisted of 400 images and lasted $18 \mathrm{~min}$ and $54 \mathrm{~s}$. 
Table I Scanning Schedule and Timeline for all Active VNS Scans

\begin{tabular}{|c|c|c|c|c|c|}
\hline Subject & TIME & HDRS & NFAD & HDRS $\times$ NFAD & Intensity \\
\hline \multirow[t]{7}{*}{ I } & I & 28 & 2 & 56 & 0.25 \\
\hline & 3 & 32 & 2 & 64 & 0.25 \\
\hline & 11 & 19 & 2 & 38 & 0.25 \\
\hline & 13 & 12 & 2 & 24 & 0.25 \\
\hline & 22 & 14 & 2 & 28 & 0.25 \\
\hline & 37 & 10 & 2 & 20 & 0.5 \\
\hline & 55 & 21 & 2 & 42 & 0.5 \\
\hline \multirow[t]{7}{*}{2} & 1 & 24 & 5 & 120 & 0.25 \\
\hline & 3 & 19 & 5 & 95 & 0.25 \\
\hline & 11 & 22 & 5 & 110 & 0.25 \\
\hline & 14 & 22 & 5 & 110 & 0.25 \\
\hline & 54 & 31 & 5 & 155 & 0.75 \\
\hline & 65 & 8 & 5 & 40 & 0.75 \\
\hline & 88 & 10 & 5 & 50 & 0.75 \\
\hline \multirow[t]{6}{*}{3} & 3 & 19 & 7 & 133 & 0.5 \\
\hline & 11 & 25 & 7 & 175 & 0.5 \\
\hline & 42 & 21 & 7 & 147 & 0.75 \\
\hline & 55 & 22 & 7 & 154 & I \\
\hline & 67 & 10 & 7 & 70 & I \\
\hline & 92 & 9 & 7 & 63 & I \\
\hline \multirow[t]{3}{*}{4} & I & 27 & 3 & 81 & 0.25 \\
\hline & 4 & 29 & 3 & 87 & 0.5 \\
\hline & 11 & 28 & 3 & 84 & 0.5 \\
\hline \multirow[t]{4}{*}{5} & 1 & 26 & 2 & 52 & 0.25 \\
\hline & 3 & 21 & 2 & 42 & 1 \\
\hline & 11 & 28 & 2 & 56 & I \\
\hline & 24 & 24 & 2 & 48 & 1 \\
\hline \multirow[t]{5}{*}{6} & 3 & 27 & 3 & 81 & 0.25 \\
\hline & 11 & 27 & 3 & 81 & 0.5 \\
\hline & 34 & 27 & 3 & 81 & 0.25 \\
\hline & 47 & 20 & 3 & 60 & 0.5 \\
\hline & 65 & 11 & 3 & 33 & 0.5 \\
\hline \multirow[t]{4}{*}{7} & 11 & 26 & 2 & 52 & 0.25 \\
\hline & 50 & 30 & 2 & 60 & 0.25 \\
\hline & 67 & 31 & 2 & 62 & 0.25 \\
\hline & 77 & 25 & 2 & 50 & 0.25 \\
\hline \multirow[t]{5}{*}{8} & 4 & 17 & 6 & 102 & 0.5 \\
\hline & 11 & 30 & 6 & 180 & 0.5 \\
\hline & 41 & 30 & 6 & 180 & 0.25 \\
\hline & 56 & 29 & 6 & 174 & 0.75 \\
\hline & 81 & 24 & 6 & 144 & 1 \\
\hline \multirow[t]{4}{*}{9} & 11 & 26 & 3 & 78 & 0.5 \\
\hline & 49 & 16 & 3 & 48 & I \\
\hline & 63 & 11 & 3 & 33 & I \\
\hline & 72 & 12 & 3 & 36 & I \\
\hline
\end{tabular}

The VNS epoch was synchronized at the beginning of each of the 10 cycles (scans 1-5). After scanning, VNS parameters were reset to those programmed when the participant reported for the scan.

\section{Data Processing Methods}

The fMRI image data were transferred to a Dell workstation, converted into Analyze format using MRICro, and analyzed with Statistical Parametric Mapping software, version 2 (Welcome Department of Imaging Neuroscience, London, UK). Data were reoriented to correspond with standard SPM format. Scans from each data set were co-registered to an image created from their mean, and corrected for motion using the standard SPM algorithm. Corrected images were reviewed for remaining motion, which was less than $0.5 \mathrm{~mm}$ along all three major axes $(x, y, z)$ for all participants. Images were timing corrected using the center slice (seven of 15) as the reference slice in an ascending sequence, with an interscan interval of $2.837 \mathrm{~s}$. Using the SPM2 algorithm, each data set was spatially normalized into Talairach space (Talairach and Tournoux, 1988) by adjusting images to conform to the SPM2 epi template through trilinear interpolation. Input and output voxel dimensions were $2 \times 2 \times 6$ and $4 \times 4 \times 4 \mathrm{~mm}$, respectively. Normalized data were spatially smoothed using a Gaussian filter width of $8 \times 8 \times 8 \mathrm{~mm}$.

Scans acquired during the placebo phase were also analyzed.

\section{Data Analysis}

Identification of voxels with statistically significant activation during VNS in individual participants ( $f$-maps using SPM2 General Linear Model; fixed effect). A pixelby-pixel bidirectional $t$-test identified areas of significantly decreased or increased response to VNS $v s$ rest (or placebo $v s$ rest), using a delayed boxcar model (10 cycles of 40 scans). In each cycle, images 1-5 were considered the VNS activation period. Images 21-23, corresponding to a period of tone stimulation, are the subject of another analysis. The remaining images, considered rest periods provided comparison with VNS. Potentially confounding motion over time was regressed out, and a high-pass filter with a cutoff period of $232 \mathrm{~s}$ was applied to remove slow signal drift.

Using all participants' f-maps, a one-sample $t$-test determined areas of significant activation or deactivation in response to VNS compared with rest periods for all participants as a group. Only clusters of activation with $Z$ values corresponding to $p<0.1$ and meeting an extended threshold of at least 15 voxels $\left(960 \mathrm{~mm}^{3}\right)$ were considered in the group analyses.

Multiple regression analysis. To investigate effects of several clinical parameters on VNS response, a multiple linear regression mixed model was designed using multiple scans from each subject and the following covariates: (1) each participant's $\mathrm{HDRS}_{24}$ at scanning $\left(\mathrm{HDRS}_{24}\right) ;(2)$ weeks since activation of VNS (TIME); (3) output current of VNS at each session, adjusted independently for clinical parameters (OUTPUT); and (4) severity of illness, based on the interaction between each participant's baseline NFAD 

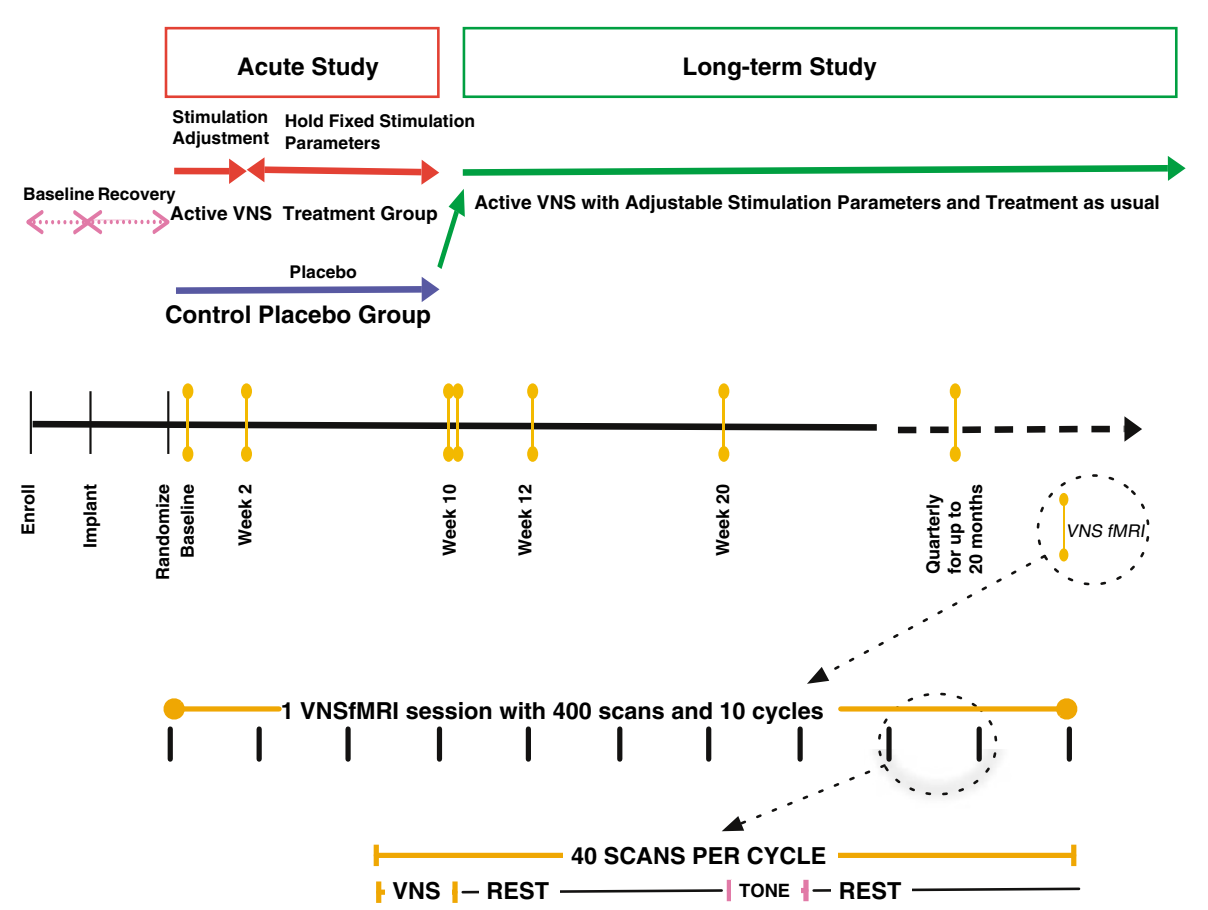

Figure I Study design and time points for serial VNS fMRI. Note the detailed a VNS fMRI scanning paradigm with 10 cycles and a total of 400 scans per session.

score and the current Hamilton Depression rating $\left(\mathrm{HDRS}_{24} \times \mathrm{NFAD}\right)$. Despite us not being able to find any references for the combined $\left(\mathrm{HDRS}_{24} \times \mathrm{NFAD}\right)$ variable, it was specifically chosen to differentiate among participants presenting with identical depression ratings but differing depressive histories and treatment resistance. Both of these variables have shown influence on clinical outcomes and thus likely to affect the brain activity and merit being considered and explored in our regression model (Sackeim, 2001; Nahas et al, 2005). Other variables likely playing an important role in the clinical outcomes to VNS therapy (ie depression sub-type, comorbid psychiatric conditions, age, specific concomitant medications, etc.) were considered but not included in this model owing to small sample sizes and unequal representation of the groups. These included gender, bipolar or unipolar depression, type of concomitant psychotropic medications. Individual participants' f-maps were entered into this analysis. Given that such an analysis would require a larger sample size than simple $t$-test to achieve the same power, only areas of activation meeting an extended threshold of a minimum of 10 voxels $\left(640 \mathrm{~mm}^{3}\right)$ and $Z$-scores equivalent $\geqslant 2.5$ were considered significant (Harrison et al, 2003).

\section{RESULTS}

In all, 107 serial scans were acquired on 17 participants. Each participant underwent a minimum of three and a maximum of seven scans. In this analysis, we report 45 active VNS scans and nine placebo scans from nine participants. Seven of these nine participants were originally randomized to placebo then switched to active VNS therapy. Fifty-three scans were not used in this analysis and included technical difficulties (eg, participant movement $>2 \mathrm{~mm}$ during scanning or poor quality, $n=26$ ), generator not restarting within scanner $(n=11)$, generator not keeping pre-set on-off duty cycle within scanner $(n=16)$, and one participant experiencing a panic attack at baseline scan and exiting this study. No patient experienced unintentional re-setting of VNS parameters into a higher range of nerve stimulation during fMRI sessions. The nine participants (six women) had a mean age, $46.8( \pm 6.2)$ years and mean duration of current episode $71.2( \pm 57.3)$ months (range, 9-194). The NFAD and VNS output current ranged from 2 to 7 (median 3 ) and 0.25 to $1.0 \mathrm{~mA}$ (median $0.5 \mathrm{~mA}$ ), respectively. Median concomitant psychotropic drugs at each scheduled timeline ranged between 3 and 5.5. All corresponding structural high resolution $\mathrm{T} 1$ images revealed no overt regional abnormalities.

\section{VNS Active Scans Group Results, Acute VNS}

When all 45 active VNS scans were grouped together (ignoring all other covariates such as time and depression state), we found significant BOLD increases during VNS in the bilateral superior temporal gyrus and left somatosensory cortex. We found significant BOLD decreases in the left middle frontal gyrus, left fusiform gyrus, left ventromedial frontal lobe, right cerebellum, and midbrain (Figure 2 and Table 2).

\section{Placebo Scans Group Results}

When nine placebo VNS scans were grouped together, we found significant BOLD increases during the no stimulation epoch compared with the rest in right orbitofrontal cortex 


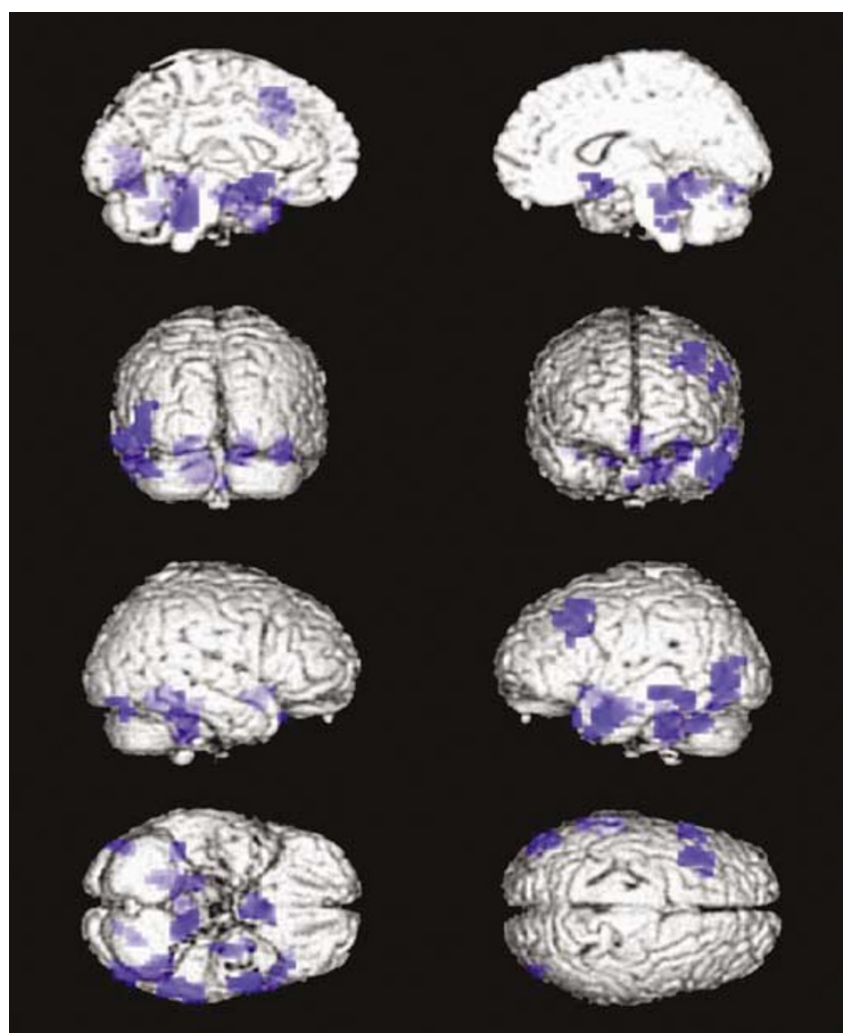

Figure 2 VNS-induced BOLD deactivations within all active 45 scans. $p=0.01$.

and right parietal cortex. Interestingly, no significant deactivations were found.

\section{Multiple Linear Regression Model}

We report a statistically significant relationship between the dependent variable, regional BOLD activation, and at least one of the independent variables in this model.

TIME. No statistically significant BOLD increases were associated with TIME.

TIME from VNS activation or exposure to VNS was associated with significant BOLD immediate VNS-induced decreases in right insula (BA 13), right medial frontal gyrus, left frontal lobe (pre- and postcentral gyrus), right temporal lobe, right parietal lobe (supramarginal gyrus), left occipital lobe (BA 17), left parietal lobe (BA 2), and left cerebellum. Figures 3 and 4 represent the linear relationship of the parameter estimates of VNS-induced activations over time. These responses were extracted from the right medial frontal gyrus $\left(\mathrm{df}=44, \mathrm{~F}=58.85, p<0.0001, r^{2}=0.577\right)$ and right insula ( $\left.\mathrm{df}=44, \mathrm{~F}=17.252, p<0.0001, r^{2}=0.286\right)$.

$H D R S_{24}$. Depressive symptoms were associated with significant BOLD increases in right temporal lobe, right insula (Brodmann area (BA) 13), and left middle frontal gyrus. Figure 5 represents the linear relationship of the parameter estimates of VNS-induced activation function of $\mathrm{HDRS}_{24}\left(\mathrm{df}=44, \mathrm{~F}=139.372, p<0.0001, r^{2}=0.76\right)$. Note: We subsequently ran the multiple regression analysis as described above with only three covariates: (1) $\mathrm{HDRS}_{24}$; (2)
TIME; and (3) OUTPUT (data not presented). At similar statistical thresholds, the results obtained from three covariates were very similar to the one summarized here with the exception of loss of right insula activation.

Depressive symptoms were associated with significant BOLD decreases in right occipital lobe (Cuneus) and right cerebellum (Pyramis).

$\mathrm{HDRS}_{24} \times$ NFAD. Severity of depressive illness was associated with significant BOLD increases in right cerebrum, occipital, right and left cerebellum.

Severity of depressive illness was associated with significant BOLD decreases in right inferior frontal gyrus, right cingulate gyrus (BA 32), left cingulate gyrus, right insula (BA 13), left middle frontal gyrus, left superior temporal gyrus, right parietal lobe (BA 7), left putamen, and left brainstem (pons).

Output current. VNS output current was associated with significant BOLD increases in left cerebellum, right parietal lobe (somatosensory BA 2), right superior frontal gyrus, and right middle frontal gyrus.

VNS output current was associated with significant BOLD decreases in left parietal lobe (precuneus), right posterior cingulate gyrus, and caudate.

\section{DISCUSSION}

This study used interleaved VNS/fMRI scanning to model dynamic regional brain responses to VNS as a function of time, the participant's depressive state, the underlying illness severity, and the stimulation output current. We found that $7 \mathrm{~s}$ trains of VNS at $20 \mathrm{~Hz}$ and $500 \mathrm{~ms}$ pulse width decreased BOLD-fMRI response in the right medial prefrontal cortex, anterior cingulate, and left anterior temporal pole and right somatosensory cortex. It also led to increased BOLD-fMRI response in the right superior temporal gyrus. These results are consistent with known vagus afferent projections (Henry, 2002; Craig, 2004) and previous VNS imaging studies(Maniker et al, 2000; Bohning et al, 2001; Lomarev et al, 2002; Mu et al, 2004). We only found BOLD increases in right orbitofrontal cortex and no significant decreases in the placebo group.

In addition to this overall analysis, which replicates and extends previous work, we performed a multiple regression model, controlling for other parameters known to influence the VNS BOLD response. To our knowledge, this type of analysis has not been previously used for VNS imaging studies. Controlling for the important covariates, we found that VNS-induced brain changes differed as a function of duration of exposure to VNS, level of depression on the study day, and VNS output current used in the scanner.

TIME since VNS device activation: our data suggest that duration of exposure to VNS accounted for most of the medial prefrontal/limbic deactivations. Even the right insula became more deactivated over time, perhaps as a function of improved clinical outcome. When participants initially received VNS, the VNS induced limbic activation. However, over time and with adjustment for other important covariates, these areas became deactivated. In Figures 4 and 5 , the linear relationship between VNS-induced 
Table 2 SPM Output/Tailarach Coordinates

\section{Condition}

Region

Active VNS group $(n=45)$

Increases with VNS compared to rest

Left superior temporal gyrus

Left somatosensory cortex (BAI)

Right superior temporal gyrus

Decreases with VNS compared to rest

Right frontal oculomotor eye field (BA8)

Right posterior cingulate gyrus (BA3I)

Right middle temporal gyrus (BA2l)

Right subgenual cingulate (BA25)

Right inferior temporal gyrus (BA37)

Right inferior temporal gyrus (BA37)

Right cerebellum

Right uncinate fasciculus

Left cerebellum

Placebo group $(n=9)$

Increases with placebo compared to rest

Right middle temporal gyrus (BA39)

Right inferior frontal gyrus (BA47)

Decreases with placebo compared to rest

Multiple linear regression model

Increases HDRS

Right temporal Lobe

Right insula (BA|3)

Left middle frontal gyrus

Decreases HDRS

Right cerebellum

Right occipital lobe

Right cerebellum

Increases time

Decreases time

Right insula (BA|3)

Right frontal lobe

Right frontal lobe

Right supramarginal gyrus

Left postcentral gyrus

Right temporal lobe

Right medial frontal gyrus

Left occipital lobe (BA 17)

Left precentral gyrus

Left cerebellum

Left postcentral gyrus (BA 2)
Tailarach coordinates $(x, y, z)$

Cluster size (n)

z-score

$\begin{array}{rrr}-56,0,0 & 182 & 4.21 \\ -36,-28,60 & 24 & 3.40 \\ 52,-16,4 & 31 & 3.19\end{array}$

$\begin{array}{rll}36,16,52 & 44 & 4.07 \\ 8,-36,-32 & 60 & 4.05 \\ 56,4,-28 & 42 & 3.90 \\ 0,8,-16 & 32 & 3.59 \\ 48,-72,0 & 41 & 3.42 \\ 56,-44,-16 & 41 & 3.32 \\ 44,-60,-32 & 18 & 3.30 \\ 28,-4,-16 & 17 & 3.13 \\ -20,-52,-32 & 23 & 3.03\end{array}$

$52,-68,16$

36

4.49

$44,40,-20$

32

4.20

$\begin{array}{rcc}20,-60,20 & 71 & 3.98 \\ 44,-4,12 & 13 & 3.15 \\ -36,4,40 & 12 & 2.86\end{array}$

$\begin{array}{rrr}24,-68,-32 & 12 & 3.22 \\ 12,-96,4 & 39 & 3.14 \\ 8,-72,-20 & 14 & 3.14\end{array}$

$\begin{array}{rrr}36,12,-4 & 134 & 3.78 \\ 24,-8,56 & 68 & 3.76 \\ 40,40,8 & 67 & 3.58 \\ 40,-48,32 & 78 & 3.40 \\ -36,-28,64 & 38 & 3.29 \\ 40,4,-24 & 19 & 3.20 \\ 8,8,52 & 26 & 3.19 \\ -16,-96,-12 & 16 & 2.97 \\ -56,8,8 & 14 & 2.86 \\ -12,-64,-32 & 14 & 2.71 \\ -56,-20,44 & 10 & 2.68\end{array}$


Table 2 Continued

\begin{tabular}{|c|c|c|c|c|}
\hline Condition & Region & Tailarach coordinates $(x, y, z)$ & Cluster size (n) & z-score \\
\hline \multicolumn{5}{|c|}{ Increases HDRS $\times$ NFAD } \\
\hline & Right cerebellum & $24,-68,-32$ & 18 & 3.2 \\
\hline & Left cerebellum & $-48,-72,-24$ & 10 & 3.07 \\
\hline & Right occipital lobe & $12,-96,4$ & 10 & 2.88 \\
\hline & Right parietal lobe (BA 7) & $12,-76,44$ & 293 & 4.29 \\
\hline & Right insula (BA |3) & $44,-4,12$ & 198 & 3.77 \\
\hline & Left middle frontal gyrus & $-36,4,40$ & 64 & 3.49 \\
\hline & Left temporal lobe & $-20,-56,16$ & 128 & 3.26 \\
\hline & Left superior temporal gyrus & $-52,-4,0$ & 10 & 2.98 \\
\hline & Right cingulate gyrus (BA 32) & $12,16,32$ & 20 & 2.59 \\
\hline & Left brainstem, pons & $-8,-16,-20$ & 10 & 2.52 \\
\hline \multicolumn{5}{|c|}{ Current output increases } \\
\hline & Left cerebellum & $-12,-64,-36$ & 16 & 3.66 \\
\hline & Right parietal lobe (BA 2) & $28,-36,64$ & 18 & 3.11 \\
\hline & Right superior frontal gyrus & $0,28,48$ & 11 & 2.90 \\
\hline & Right middle frontal gyrus & $20,-12,60$ & 12 & 2.83 \\
\hline \multicolumn{5}{|c|}{ Current output decreases } \\
\hline
\end{tabular}

Z-sore $>2.5$.

parameters estimates became predominantly negative after week 30. This is also the time when pronounced clinical improvements in symptoms take place.

Depression level on scan day: the treatment resistance of each participant's depression helps explain the larger but complementary deactivation of medial and lateral prefrontal cortex and left superior temporal gyrus, whereas depressive symptoms alone contribute to activation of the right insula. This is important given the insula's role in viceroautonomic and limbic function, and in somatic pain (Craig, 2003). Also of interest is the dynamic switch in the right insula's response to VNS. Acute VNS appears to primarily deactivate the right insula in mild-to-moderate depression but correlate with higher activation in relationship to increased severity of depressive symptoms (see Figure 6). This reversal in response may be in keeping with known anatomy of the vagus nerve and afferent parasympathetic pathways that provide sensory inputs to a hierarchically integrative network that extends to the insula via the thalamus and ultimately provides a means of 'introceptive' representations (Craig, 2004). It also provides a hypothesis explaining why VNS output current has been substantially lower in depression than epilepsy studies, despite an attempt in all studies to increase the VNS output intensity to the highest tolerated dose. Among participants with greater depression, VNS produces more activation in the right insula, an integrative center for pain perception. Depressed patients may perceive greater pain than epilepsy patients receiving VNS at the same parameter settings.

VNS output current: the patient's subjective perception of VNS greatly depends on the stimulation parameters, particularly the intensity of the stimulation (Sackeim et al, 2001). The increased activity in the right somatosensory area (likely neck and throat) associated with higher VNS output current provides an internal quality control. In addition, greater output current is associated with increased activity in the right middle frontal gyrus and decreased activity in the posterior cingulate.

\section{Access to Mood-Regulating Networks}

The vagus nerve, classically described as the 'wandering nerve', sends signals from the central nervous system to control the peripheral cardiovascular, respiratory, and 


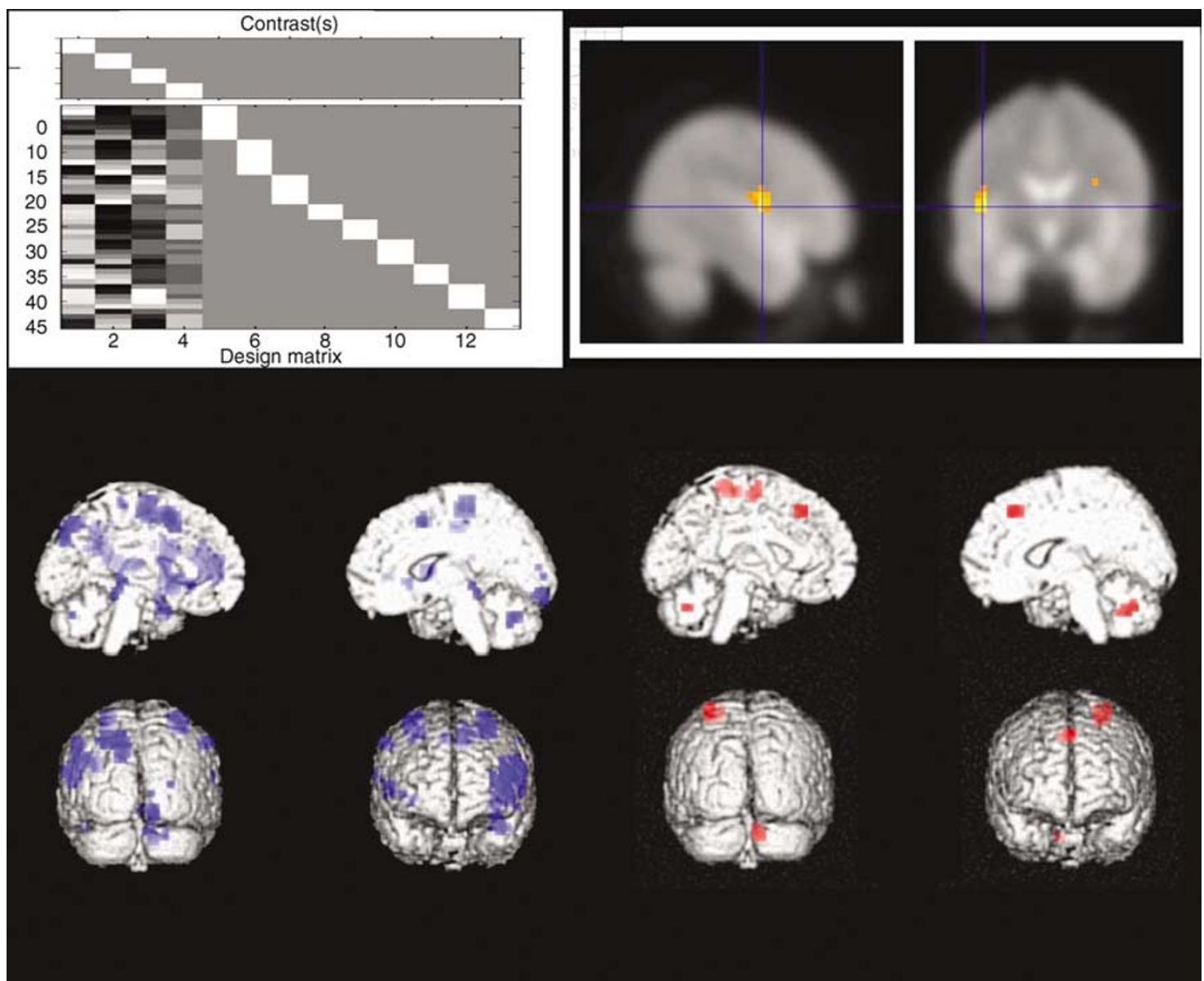

Figure 3 Design Matrix (top left) of the multiple regression model used and selected independent variables BOLD contrasts including: increases in insula associated with $\mathrm{HDRS}_{24}$ (top right), decreases in medial prefrontal and limbic structures associated with TIME of Exposure to VNS therapy (bottom left) and increases in right somatosensory cortex associated with intensity of stimulation (bottom right).

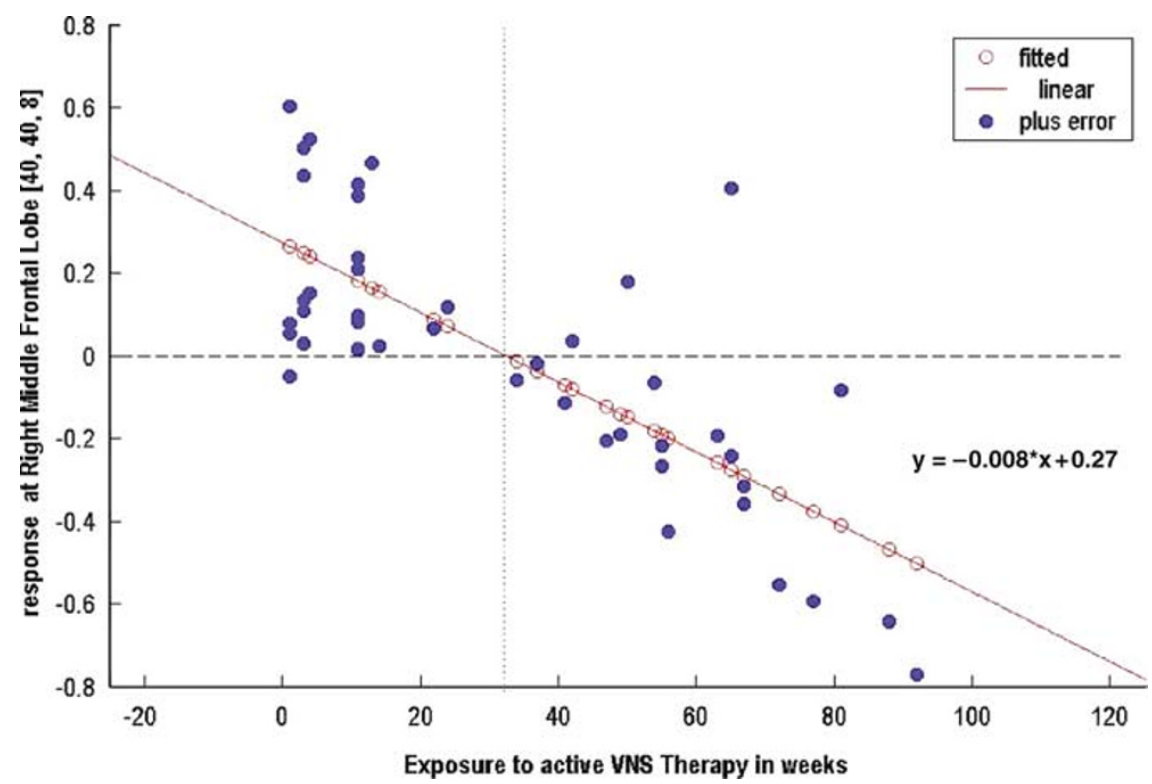

Figure 4 Relationship between VNS-induced parameters estimates response in the right medial prefrontal gyrus and exposure to active VNS therapy in weeks $\left(d f=44, F=58.85, p<0.000 I, r^{2}=0.577\right)$. Note that the linear response becomes predominantly negative after week 30 .

gastrointestinal systems. However, $80 \%$ of its fibers are afferent, carrying information from the viscera to the brain (Foley and DuBois, 1937). The fibers enter the midbrain at the nucleus tractus solitaris (NTS) level. From the midbrain, they either loop back to the periphery in a reflex arc, connect to the reticular activating system, or reach the parabrachial nucleus and its connections to the NTS, raphe nucleus, locus ceruleus (LC), the thalamus, paralimbic, 


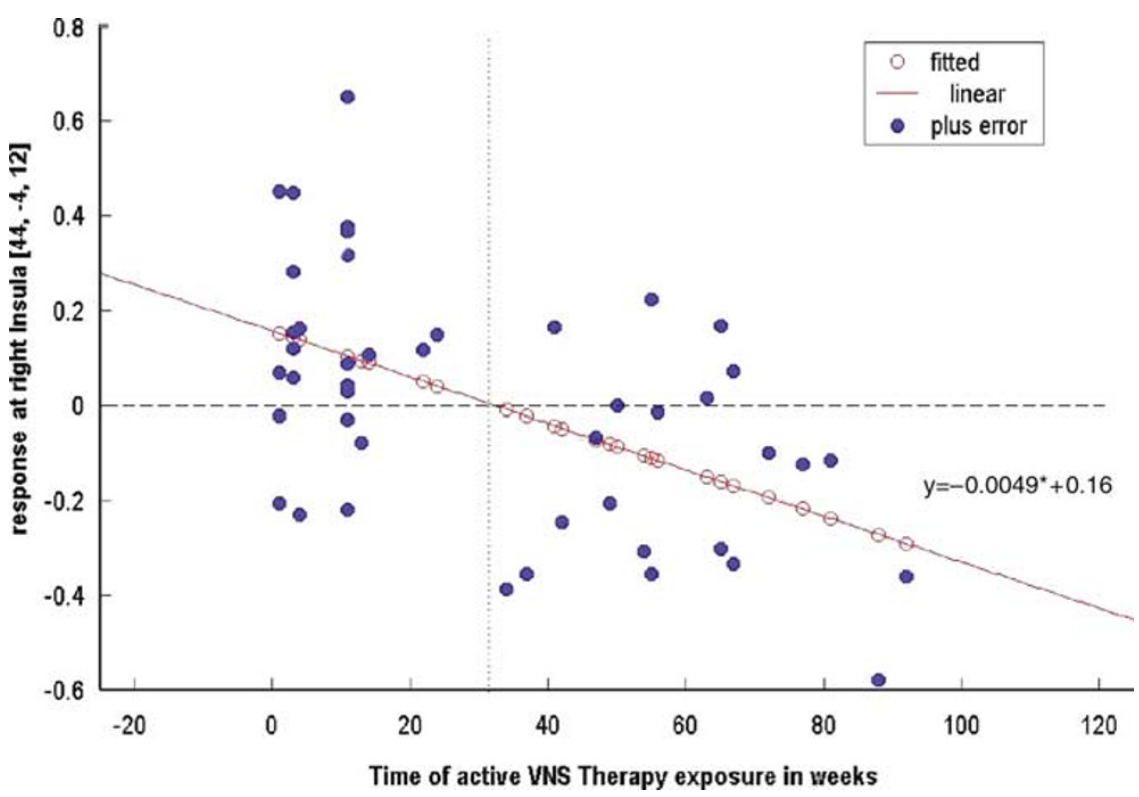

Figure 5 Relationship between VNS-induced parameters estimates response in the right insula and exposure to active VNS therapy in weeks (df $=44$, $\left.F=17.252, p<0.000 I, r^{2}=0.286\right)$. Note that here again, the linear response becomes predominantly negative after week 30.

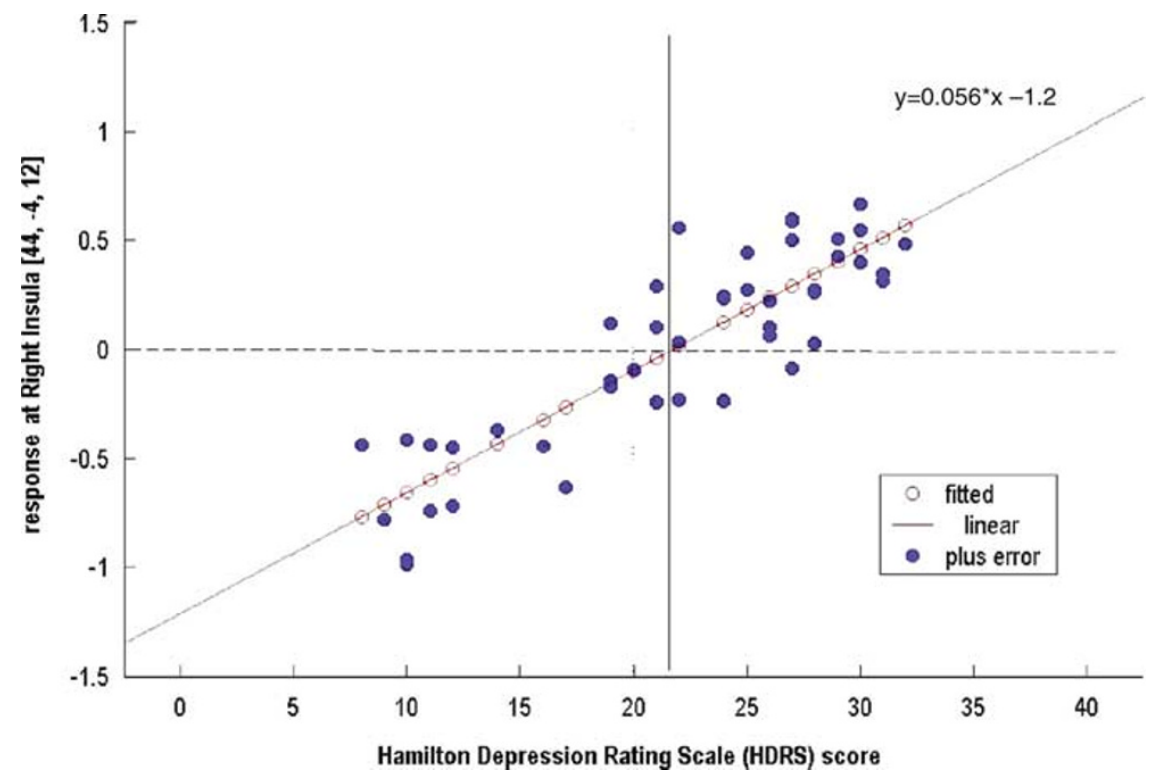

Figure 6 Relationship between VNS-induced parameters estimates response in the right insula and the depressive symptoms of each individual at the time of scanning as measured by HDRS $\left(\mathrm{df}=44, \mathrm{~F}=139.372, p<0.000 \mathrm{I}, \mathrm{r}^{2}=0.76\right)$. Note that the linear response becomes predominantly positive after a HDRS score of 22 .

limbic, and cortical regions, including anterior insula and cingulate cortex (Hallowitz and MacLean, 1977). VNS modulates brain function through this route (Henry, 2002). The interplay between VNS and central nervous system monoamines has been demonstrated (Ben-Menachem et al, 1995; Naritoku et al, 1995; Krahl et al, 1998; Carpenter et al, 2004). The brainstem evidences specific acute markers of neuronal activity in vagus nerve nuclei, LC noradrenergic nuclei, and cochlear nucleus (Naritoku et al, 1995). Lesioning the LC interferes with the antiepileptic effect in rodents (Krahl et al, 1998). VNS induces specific nuclear fos immunolabeling in several forebrain structures, including the posterior cortical amygdaloid nucleus and cingulate retrosplenial cortex (Naritoku et al, 1995). Conversely, cerebral spinal fluid (CSF) studies involving epilepsy patients reveal increased $\gamma$-aminobutyric acid (GABA) as well as homovanillic acid (HVA), and 5hydroxyindoleacetic acid (5-HIAA), the major metabolites of dopamine and serotonin, respectively, and decreased glutamate after 3 months of treatment (Ben-Menachem et al, 1995). After 24 weeks in depressed subjects, VNS caused increases in CSF HVA but not in mean concentration of NE, 5-HIAA, 3-methoxy-4-hydroxyphenylglycol, or GABA (Carpenter et al, 2004). 


\section{Comparison with Other Imaging Studies of Antidepressant Treatments}

The prefrontal/limbic pattern of activations observed in our study supports this general neuroanatomic framework. Moreover, VNS-induced effects are largely consistent with other published investigations of antidepressant mechanisms of action. Much of this literature has focused primarily on exploring pharmacologic interventions and their postulated effects on mood-regulating brain networks. Chronic antidepressant drug treatment, such as a serotonin reuptake inhibitors (SSRI) like sertraline (Buchsbaum et al, 1997; Nobler et al, 2000; Drevets et al, 2002), fluoxetine (Mayberg et al, 2000), or paroxetine (Brody et al, 2001), seems to reduce metabolism in the limbic areas and/or ventral ACC of subjects showing a persistent, positive treatment response. Clinical remission was also associated with decreased ACC activity in a study of subjects receiving either an SSRI or a norepinephrine-serotonin reuptake inhibitor (NSRI) (Holthoff et al, 2004), although these two classes of antidepressants may not share identical effects on brain metabolism.

Other brain stimulation modalities for treating depression have also shown dynamic modulation of prefrontal/limbic regions. ECT's antidepressant effect focuses on the dynamic interplay between the ictal and post-ictal phases (Rosenberg et al, 1988). During the ictal period, cerebral blood flow increases up to $300 \%$ of baseline and cerebral metabolic rate up to 200\%; these measures decrease post-ictally. The degree of prefrontal and medial frontal deactivation immediately after ECT correlates with later clinical improvement. This inverse relationship holds true 2 months after ECT (Nobler et al, 2001). TMS, when applied repetitively over the prefrontal cortex, has also shown antidepressant effects (Kozel and George, 2002; Gershon et al, 2003) and is associated with local and transynaptic distal modulation of subcortical regions, including ACC and amygdala (Teneback et al, 1999; Speer et al, 2000; Nahas et al, 2001b; Strafella et al, 2001). Its antidepressive properties may also depend on the severity of underlying depression (Teneback et $a l, 1999$ ) and the stimulation parameters (Kimbrell et al, 1999; Nahas et al, 2001b). A realtime assessment of brain activity with prefrontal interleaved TMS fMRI (Bohning et al, 1998) in a depressed cohort with concomitant medications, very similar to our cohort, has shown medial prefrontal deactivation (Li et al, 2003) not seen in healthy volunteers (Nahas et al, 2001a). Finally, an open study has reported antidepressant benefits of DBS posterior to the subgenual cingulate with concurrent deactivation of that region (Mayberg et al, 2005).

\section{Progressive Adaptation}

The deactivations of medial prefrontal cortex with VNS are similar to other antidepressant treatments, but they also suggest adaptation over time. Unlike pharmacologic interventions evidencing antidepressant effects after a few weeks of treatment (Mayberg et al, 2000), VNS clinical antidepressant effects occur later, after several months. The turning point for right insula, a key region in explicit subjective awareness (Critchley et al, 2004), and medial prefrontal activations seems to occur around 30 weeks of active VNS. If replicated in future studies, this particular characteristic may reflect VNS' relatively delayed time of effect. Of interest is a recent SPECT study showing limbic deactivations with only 4 weeks of VNS therapy (Zobel et al, 2005). Given the rapid response rate seen in this European study, the treatment resistance level may play an important role in how quickly VNS modulates these networks. In addition, SPECT and fMRI modalities differ in time their respective time resolutions and thus may explain some of the divergent results. Effective and progressive modulation of key brain regions may ultimately explain this distinctive therapeutic feature. Another distinctive clinical feature awaiting replication is the prolonged response and fewer relapses of depressed participants receiving adjunctive VNS (Nahas et al, 2005). Although our data do not fully explain this phenomenon, the progressive adaptation of crucial neuronal networks may complement animal epilepsy models in which VNS confers chronic progressive prophylactic effects, with seizure counts reduced more after chronic stimulation than after acute stimulation over less than a day. Similar research is needed in animal depression models.

\section{Limitations}

The 'magnetic switch' designed to allow epilepsy patients to self-administer an extra train of stimulation to help curb a full-blown seizure has limited our ability to successfully scan all participants. Despite a customized surgical implantation rotating the device about $45^{\circ}$ counterclockwise to allow generator reactivation once the participant is parallel to the main magnetic field of the scanner, the VNS generator restarted in the scanner during only two-thirds of the sessions. This difficulty affected the statistical power of this study. Additionally, this fMRI paradigm had a relatively brief active-VNS epoch. The small number of participants with bipolar depression in the study precluded specific analysis differentiating MDD from BPAD responses. Given the adjunctive nature of this therapy, controlling for concomitant psychotropic medications is a limitation to this study. The median number was, however, relatively stable across the length of the study. Finally, the correlations presented here were derived from nine subjects but a total of 45 scans. And although this the largest imaging study reported in VNS literature, this does limit the interpretation of these data.

In conclusion, VNS/fMRI seems useful in studying the effects of VNS on brain activity both acutely and over time. VNS/fMRI may even allow in the future tailoring of stimulation parameters to optimally modulate specific regions and study progressive therapeutic adaptations. Although VNS and other antidepressant interventions share several general similarities, much work remains to fully elucidate the VNS mechanisms of actions.

\section{ACKNOWLEDGEMENTS}

Grant support was received from Cyberonics Inc. (DEB), The Dana Foundation (DEB), The Stanley Foundation (CT) and a grant in kind from the Center for Advanced Imaging Research (CAIR) at MUSC. Other support includes NIMH 
K08 MH070615-01A1 (ZN), NIMH R21 MH065630-01 (CM, ZN), NIMH R01 MH069896-01 A1 (MSG, SK, BA, CM, ZN), NINDS R01 NS40956-01 (DEB, MSG, ZN, JW). ZN and MSG are paid consultants to CYBX. MSG is a member of CYBX Mechanism of Action and Depression Advisory Boards.

\section{REFERENCES}

Barnes A, Duncan R, Chisholm JA, Lindsay K, Patterson J, Wyper D (2003). Investigation into the mechanisms of vagus nerve stimulation for the treatment of intractable epilepsy, using 99mTc-HMPAO SPET brain images. Eur J Nucl Med $\mathrm{Mol}$ Imaging 30: 301-305.

Ben-Menachem E, Hamberger A, Hedner T, Hammond EJ, Uthman BM, Slater J et al (1995). Effects of vagus nerve stimulation on amino acids and other metabolites in the CSF of patients with partial seizures. Epilepsy Res 20: 221-227.

Bohning DE, Lomarev MP, Denslow S, Nahas Z, Shastri A, George MS (2001). Feasibility of vagus nerve stimulation-synchronized blood oxygenation level-dependent functional MRI. Invest Radiol 36: 470-479.

Bohning DE, Shastri A, Nahas Z, Lorberbaum JP, Anderson SW, Dannels W et al (1998). Echoplanar BOLD fMRI of brain activation induced by concurrent transcranial Magnetic stimulation (TMS). Invest Radiol 33: 336-340.

Brody AL, Saxena S, Stoessel P, Gillies LA, Fairbanks LA, Alborzian S et al (2001). Regional brain metabolic changes in patients with major depression treated with either paroxetine or interpersonal therapy: preliminary findings. Arch Gen Psychiatry 58: 631-640.

Buchsbaum MS, Wu J, Siegel BV, Hackett E, Trenary M, Abel L et al (1997). Effect of sertraline on regional metabolic rate in patients with affective disorder. Biol Psychiatry 41: 15-22.

Carpenter LL, Moreno FA, Kling MA, Anderson GM, Regenold WT, Labiner DM et al (2004). Effect of vagus nerve stimulation on cerebrospinal fluid monoamine metabolites, norepinephrine, and gamma-aminobutyric acid concentrations in depressed patients. Biol Psychiatry 56: 418-426.

Chae JH, Nahas Z, Lomarev M, Denslow S, Lorberbaum JP, Bohning DE et al (2003). A review of functional neuroimaging studies of vagus nerve stimulation (VNS). J Psychiatr Res 37: 443-455.

Craig AD (2003). Pain mechanisms: labeled lines versus convergence in central processing. Annu Rev Neurosci 26: 1-30.

Craig AD (2004). Human feelings: why are some more aware than others? Trends Cogn Sci 8: 239-241.

Critchley HD, Wiens S, Rotshtein P, Ohman A, Dolan RJ (2004). Neural systems supporting interoceptive awareness. Nat Neurosci 7: 189-195.

Drevets WC, Bogers W, Raichle ME (2002). Functional anatomical correlates of antidepressant drug treatment assessed using PET measures of regional glucose metabolism. Eur Neuropsychopharmacol 12: 527-544.

Ebert D, Feistel H, Barocka A (1991). Effects of sleep deprivation on the limbic system and the frontal lobes in affective disorders: a study with Tc-99m-HMPAO SPECT. Psychiatry Res 40: 247-251.

Ebert D, Feistel H, Barocka A, Kaschka W (1994). Increased limbic flow and total sleep deprivation in major depression with melancholia. Psychiatry Res 55: 101-109.

Fagiolini A, Kupfer DJ (2003). Is treatment-resistant depression a unique subtype of depression? Biol Psychiatry 53: 640-648.

Foley JO, DuBois F (1937). Quantitative studies of the vagus nerve in the cat. I. The ratio of sensory and motor studies. J Comp Neurol 67: 49-67.

Garnett ES, Nahmias C, Scheffel A, Firnau G, Upton AR (1992). Regional cerebral blood flow in man manipulated by direct vagal stimulation. Pacing Clin Electrophysiol 15: 1579-1580.
George MS, Rush AJ, Marangell LB, Sackeim HA, Brannan SK, Davis SM et al (2005). A one-year comparison of vagus nerve stimulation with treatment as usual for Treatment-resistant depression. Biol Psychiatry 58: 364-373.

Gershon AA, Dannon PN, Grunhaus L (2003). Transcranial magnetic stimulation in the treatment of depression. Am J Psychiatry 160: 835-845.

Goldapple K, Segal Z, Garson C, Lau M, Bieling P, Kennedy S et al (2004). Modulation of cortical-limbic pathways in major depression: treatment-specific effects of cognitive behavior therapy. Arch Gen Psychiatry 61: 34-41.

Hallowitz RA, MacLean PD (1977). Effects of vagal volleys on units of intralaminar and juxtalaminar thalamic nuclei in monkeys. Brain Res 130: 271-286.

Hamilton M (1967). Development of a rating scale for primary depressive illness. Br J Soc Clin Psychol 6: 278-296.

Harrison L, Penny WD, Friston K (2003). Multivariate autoregressive modeling of fMRI time series. Neuroimage 19: $1477-1491$.

Henry TR (2002). Therapeutic mechanisms of vagus nerve stimulation. Neurology 596 Suppl 4: S3-S14.

Henry TR, Bakay RA, Pennell PB, Epstein CM, Votaw JR (2004). Brain blood-flow alterations induced by therapeutic vagus nerve stimulation in partial epilepsy: II. Prolonged effects at high and low levels of stimulation. Epilepsia 45: 1064-1070.

Henry TR, Bakay RA, Votaw JR, Pennell PB, Epstein CM, Faber TL et al (1998). Brain blood flow alterations induced by therapeutic vagus nerve stimulation in partial epilepsy: I. Acute effects at high and low levels of stimulation. Epilepsia 39: 983-990.

Henry TR, Votaw JR, Pennell PB, Epstein CM, Bakay RA, Faber TL et al (1999). Acute blood flow changes and efficacy of vagus nerve stimulation in partial epilepsy. Neurology 52: $1166-1173$

Holthoff A, Beuthien-Baumann B, Zundorf G, Triemer A, Ludecke S, Winiecki $\mathrm{P}$ et al (2004). Changes in brain metabolism associated with remission in unipolar major depression. Acta Psychiatr Scand 110: 184-194.

Kimbrell TA, Little JT, Dunn RT, Frye MA, Greenberg BD, Wassermann EM et al (1999). Frequency dependence of antidepressant response to left prefrontal repetitive transcranial magnetic stimulation (rTMS) as a function of baseline cerebral glucose metabolism. Biol Psychiatry 46: 1603-1613.

Kleinschmidt A, Bruhn H, Kruger G, Merboldt KD, Stoppe G, Frahm J (1999). Effects of sedation, stimulation, and placebo on cerebral blood oxygenation: a magnetic resonance neuroimaging study of psychotropic drug action. NMR Biomed 12: 286-292.

Ko D, Heck C, Grafton S, Apuzzo ML, Couldwell WT, Chen T et al (1996). Vagus nerve stimulation activates central nervous system structures in epileptic patients during PET H2(15)O blood flow imaging. Neurosurgery 39: 426-430; discussion 430-431.

Kozel FA, George MS (2002). Meta-analysis of left prefrontal repetitive transcranial magnetic stimulation (rTMS) to treat depression. Psychiatric Practice 8: 270-275.

Krahl SE, Clark KB, Smith DC, Browning RA (1998). Locus coeruleus lesions suppress the seizure-attenuating effects of vagus nerve stimulation. Epilepsia 39: 709-714.

Li X, Nahas Z, Anderson B, Kozel AF, Yamanaka K, Bohning DE et al (2003). Transcranial magnetic stimulation delivered over the left prefrontal cortex of depressed patients increases local cortical activity as well as in the right orbitofrontal cortex, hippocampus, insulan and thalamus. Biol Psychiatry 53: 541.

Liu WC, Mosier K, Kalnin AJ, Marks D (2003). BOLD fMRI activation induced by vagus nerve stimulation in seizure patients. J Neurol Neurosurg Psychiatry 74: 811-813.

Lomarev M, Denslow S, Nahas Z, Chae JH, George MS, Bohning DE (2002). Vagus nerve stimulation (VNS) synchronized BOLD 
fMRI suggests that VNS in depressed adults has frequency/dose dependent effects. J Psychiatr Res 36: 219-227.

Maniker A, Liu WC, Marks D, Moser K, Kalnin A (2000). Positioning of vagal nerve stimulators: technical note. Surg Neurol 53: 178-181.

Marangell LB, Rush AJ, George MS, Sackeim HA, Johnson CR, Husain MM et al (2002). Vagus nerve stimulation (VNS) for major depressive episodes: one year outcomes. Biol Psychiatry 51: 280-287.

Mayberg HS, Brannan SK, Tekell JL, Silva JA, Mahurin RK, McGinnis S et al (2000). Regional metabolic effects of fluoxetine in major depression: serial changes and relationship to clinical response. Biol Psychiatry 48: 830-843.

Mayberg HS, Lozano AM, Voon V, McNeely HE, Seminowicz D, Hamani C et al (2005). Deep brain stimulation for treatmentresistant depression. Neuron 45: 651-660.

$\mathrm{Mu} \mathrm{Q}$, Bohning DE, Nahas Z, Walker J, Anderson B, Johnson KA et al (2004). Acute vagus nerve stimulation using different pulse widths produces varying brain effects. Biol Psychiatry 55: 816-825.

Nahas Z, Lomarev M, Roberts DR, Shastri A, Lorberbaum JP, Teneback C et al (2001a). Unilateral left prefrontal transcranial magnetic stimulation (TMS) produces intensity-dependent bilateral effects as measured by interleaved BOLD fMRI. Biol Psychiatry 50: 712-720.

Nahas Z, Marangell LB, Husain MM, Rush AJ, Sackeim HA, Lisanby SH et al (2005). Two-year outcome of vagus nerve stimulation (VNS) for treatment of major depressive episodes. J Clin Psychiatry 66: 1097-1104.

Nahas Z, Teneback CC, Kozel A, Speer AM, DeBrux C, Molloy M et al (2001b). Brain effects of TMS delivered over prefrontal cortex in depressed adults: role of stimulation frequency and coilcortex distance. J Neuropsychiatry Clin Neurosci 13: 459-470.

Narayanan JT, Watts R, Haddad N, Labar DR, Li PM, Filippi CG (2002). Cerebral activation during vagus nerve stimulation: a functional MR study. Epilepsia 43: 1509-1514.

Naritoku DK, Terry WJ, Helfert RH (1995). Regional induction of Fos immunoreactivity in the brain by anticonvulsant stimulation of the vagus nerve. Epilepsy Res 22: 53-62.

Nobler MS, Oquendo MA, Kegeles LS, Malone KM, Campbell CC, Sackeim HA et al (2001). Decreased regional brain metabolism after ect. Am J Psychiatry 158: 305-308.

Nobler MS, Roose SP, Prohovnik I, Moeller JR, Louie J, Van Heertum RL et al (2000). Regional cerebral blood flow in mood disorders, V: effects of antidepressant medication in late-life depression. Am J Geriatr Psychiatry 8: 289-296.

O’Reardon JP, Amsterdam JD (1998). Treatment-resistant depression: progress and limitations. Psychiatr Ann 28: 633-640.

Prudic J, Olfson M, Marcus SC, Fuller RB, Sackeim HA (2004). Effectiveness of electroconvulsive therapy in community settings. Biol Psychiatry 55: 301-312.

Ring HA, White S, Costa DC, Pottinger R, Dick JP, Koeze T et al (2000). A SPECT study of the effect of vagal nerve stimulation on thalamic activity in patients with epilepsy. Seizure 9: 380-384.

Rosenberg R, Vorstrup S, Andersen A, Bolwig T (1988). Effect of ECT on Cerebral Blood Flow Assessed with SPECT. Convulsive Ther 4: 62-73.
Rush AJ, George MS, Sackeim HA, Marangell LB, Husain MM, Giller C et al (2000). Vagus nerve stimulation (VNS) for treatment-resistant depressions: a multicenter study. Biol Psychiatry 47: 276-286.

Rush AJ, Marangell LB, Sackeim HA, George MS, Brannan SK, Davis SM et al (2005a). Vagus nerve stimulation for treatmentresistant depression: a randomized, controlled acute phase trial. Biol Psychiatry 58: 347-354.

Rush AJ, Sackeim HA, Marangell LB, George MS, Brannan SK, Davis SM et al (2005b). Effects of 12 months of vagus nerve stimulation in treatment-resistant depression: a naturalistic study. Biol Psychiatry 58: 355-363.

Sackeim HA (2001). The definition and meaning of treatmentresistant depression. J Clin Psychiatry 62(Suppl 16): 10-17.

Sackeim HA, Rush AJ, George MS, Marangell LB, Husain MM, Nahas $\mathrm{Z}$ et al (2001). Vagus nerve stimulation (VNS) for treatment-resistant depression: efficacy, side effects, predictors of outcome. Neuropsychopharmacology 25: 713-728.

Speer AM, Kimbrell TA, Wasserman EM, Repella J, Wilis MW, Hersovitch $\mathrm{P}$ et al (2000). Opposite effects of high and low frequency rTMS on regional brain activity in depressed patients. Biol Psychiatry 48: 1133-1141.

Strafella AP, Paus T, Barrett J, Dagher A (2001). Repetitive transcranial magnetic stimulation of the human prefrontal cortex induces dopamine release in the caudate nucleus. J Neurosci 21: RC157.

Sucholeiki R, Alsaadi TM, Morris III GL, Ulmer JL, Biswal B, Mueller WM (2002). fMRI in patients implanted with a vagal nerve stimulator. Seizure 11: 157-162.

Talairach J, Tournoux P (1988). Co-Planar Stereotaxic Atlas of the Human Brain: 3-Dimensional Proportional System: An Approach to Cerebral Imaging. Thieme: New York.

Teneback CC, Nahas Z, Speer AM, Molloy M, Stallings LE, Spicer KM et al (1999). Changes in prefrontal cortex and paralimbic activity in depression following two weeks of daily left prefrontal TMS. J Neuropsychiatry Clin Neurosci 11: 426-435.

Van Laere K, Vonck K, Boon P, Versijpt J, Dierckx R (2002). Perfusion SPECT changes after acute and chronic vagus nerve stimulation in relation to prestimulus condition and long-term clinical efficacy. J Nucl Med 43: 733-744.

Vonck K, Boon P, Van Laere K, D’Have M, Vandekerckhove T, O'Connor $S$ et al (2000). Acute single photon emission computed tomographic study of vagus nerve stimulation in refractory epilepsy. Epilepsia 41: 601-609.

Wu JC, Bunney WE (1990). The biological basis of an antidepressant response to sleep deprivation and relapse: review and hypothesis. Am J Psychiatry 147: 14-21.

Wu JC, Gillin JC, Buchsbaum MS, Hershey T, Johnson JC, Bunney WE (1992). Effect of sleep deprivation on brain metabolism of depressed patients. Am J Psychiatry 149: 538-543.

Zobel A, Joe A, Freymann N, Clusmann H, Schramm J, Reinhardt $M$ et al (2005). Changes in regional cerebral blood flow by therapeutic vagus nerve stimulation in depression: an exploratory approach. Psychiatry Res 139: 165-179. 